\title{
Direct Application of Kantorovich's Method in BVP
}

\author{
J. Evangeline Cicelia* \\ Department of Mathematics, Bharath Institute of Science \& Technology, \\ Bharath University, Selaiyur, Chennai -600073, Tamil Nadu, India; evangelinecicelia@gmail.com
}

\section{Abstract}

In this article a new approach of finding approximate solution as well as comparing it with its exact solution is discussed. By using Kantorovich's method, we get approximate solution for the Boundary Value Problems (BVP) to be extremized. Also, we compare it with exact solution. This method is explained by suitable example problem.

Keywords: Approximate Solution, Exact Solution, Kantorovich's Method, Boundary Value Problems (BVP)

\section{Introduction}

When the extremum of the functional

$$
\begin{aligned}
& J[y(x)]=\int_{x_{1}}^{x_{2}} F\left(x, y, y^{\prime}\right) d x \\
& y\left(x_{1}\right)=y_{1}, \quad y\left(x_{2}\right)=y_{2} \text { is sought, the probable }
\end{aligned}
$$
conditions are:

1. The function $F(x, y, z)$ is continuous with respect to all the arguments for any $z$ and for $(x, y) \in D$ where $D$ is a closed domain of the XY-plane in which lie the curves $y_{\mathrm{n}}(x)$.

2. There exist constants $\alpha>0, p>1, \beta$ for which $F(x, y, z) \geq \alpha|Z|^{p}+\beta$

no matter what $z$ is and for any point. $(x, y) \in D$

3 . The function $F(x, y, z)$ has a continuous partial derivative $F_{z}(x, y, z)$; for any point $(x, y) \in D$ this derivative is a non-decreasing function of $z(-\infty<z<+\infty)$.

The foregoing conditions are, in particular, fulfilled for functionals of the form

$$
\begin{aligned}
& J[y]=\int_{x_{1}}^{x_{2}}\left[p(x) y^{\prime 2}+q(x) y^{2}+2 r(x) y\right] d x, \\
& y\left(x_{1}\right)=a, y\left(x_{2}\right)=b
\end{aligned}
$$

where, $p(x), q(x), r(x)$ are given functions continuous on $\left[x_{1}, x_{2}\right], p(x)$ has continuous derivative $p^{\prime}(x)$, and $p(x)>0$, $q(x) \geq 0$.
If this method is used to determine the absolute extremum of the functional, then the approximate value of the minimum of the functional is obtained with an excess and that of the maximum, with a deficit. The successful use of this method depends largely on how apt the choice is of the system of coordinate functions $\left\{\varphi_{i}(x)\right\}$.

In many cases, it suffices to take a linear combination of two or three functions $\varphi_{i}(x)$ in order to obtain quite satisfactory an approximation to the exact solution.

In finding the approximate extremum of functionals $J\left[z\left(x_{1}, x_{2}, \ldots x_{\mathrm{n}}\right)\right]$ that depend on functions of several independent variables, a coordinate system of functions is chosen:

$$
\varphi_{1}\left(x_{1}, x_{2}, \ldots x_{n}\right), \varphi_{2}\left(x_{1}, x_{2}, \ldots x_{n}\right), \ldots \ldots \varphi_{m}\left(x_{1}, x_{2}, \ldots x_{n}\right), \ldots \ldots
$$

An approximate solution of the variation problem is sought in the form

$$
z_{m}=\sum_{k=1}^{m} \alpha_{k} \varphi_{n}\left(x_{1}, x_{2}, \ldots x_{n}\right)
$$

where, the coefficient $\alpha_{k}$ are certain constant numbers. To determine them, we proceed as before and form a system of equations $\frac{\partial \phi}{\partial \alpha_{k}}=0(k=1,2, \ldots, n)$, where $\phi\left(\alpha_{1}\right.$, $\left.\alpha_{2}, \ldots \ldots \alpha_{n}\right)$ is the result of substituting $z_{m}$ into the functional $J[z]$.

This method can be illustrated by the following solved problem.

*Author for correspondence 
1.1 Problem: Find the approximate solution to the problem of the minimum of the functional

$$
\begin{gathered}
J[y]=\int_{0}^{1}\left(y^{\prime^{2}}-y^{2}+2 x y\right) d x, \\
y(0)=y(1)=0
\end{gathered}
$$

and compare it with the exact solution.

Solution: We choose the system of coordinate functions $\varphi_{k}(x)$ thus:

$$
\varphi_{k}(x)=(1-x) x^{k}(k=1,2, \ldots \ldots)
$$

The function $\varphi_{k}(x)$ clearly satisfying the boundary conditions $\varphi_{k}(0)-\varphi_{k}(1)=0$ are linearly independent, and constitute a complete system in the space $\mathrm{C}_{1}[0,1]$.

For $k=1$ we get $y_{1}(x)=\alpha_{1}\left(x-x^{2}\right)$ substituting this expression for $y_{1}(x)$ into the functional (1), we get

$$
\begin{aligned}
J\left[y_{1}(x)\right] & =\int_{0}^{1}\left[\alpha_{1}^{2}(1-2 x)^{2}-\alpha_{1}^{2}\left(x-x^{2}\right)^{2}+2 \alpha_{1} x\left(x-x^{2}\right)\right] d x \\
& =\int_{0}^{1}\left[\alpha_{1}^{2}\left(1-4 x+4 x^{2}-x^{2}+2 x^{3}-x^{4}\right)+2 \alpha_{1}\left(x^{2}-x^{3}\right)\right. \\
& =\frac{3}{10} \alpha_{1}^{2}+\frac{1}{6} \alpha_{1}=\phi\left(\alpha_{1}\right)
\end{aligned}
$$

The coefficient $\alpha_{1}$ is found from the equation

$$
\frac{\partial \phi}{\partial \alpha_{1}}=\frac{3}{5} \alpha_{1}+\frac{1}{6}=0
$$

where $\alpha_{1}=-\frac{5}{18}$. Consequently,

$y_{1}(x)=-\frac{5}{18} x-\frac{5}{18} x^{2}$ Exact solution: Euler's equation for the given functional is

$$
y^{\prime \prime}+y=x
$$

Solving this inhomogeneous linear equation, we find

$$
y(x)=C_{1} \cos x+C_{2} \sin x+x
$$

Using boundary condition $y(0)-y(1)=0$ we finally get

$$
y_{1}(x)=x-\frac{\sin x}{\sin 1}
$$

Let us compare the exact solution with the approximate one:

\begin{tabular}{ccc}
\hline $\mathbf{x}$ & $\begin{array}{l}\text { Exact } \\
\text { Solution }\end{array}$ & $\begin{array}{l}\text { Approximate } \\
\text { solution }\end{array}$ \\
\hline 0.00 & 0 & 0 \\
0.25 & -0.044 & -0.052 \\
0.50 & -0.070 & -0.069 \\
0.75 & -0.060 & -0.052 \\
1.00 & 0 & 0 \\
\hline
\end{tabular}

RESULT: Hence proved.

\section{Conclusion}

This paper facilitates to solve boundary value problems, in finding approximate solution and comparing it with its exact solution by using Kantorovich's method.

\section{Bibliography}

1. Bardaro C, Butzer PL, Stens RL, Vinti G. Kantorovich-type generalized sampling series in the setting of Orlicz spaces. Sampl Theory Signal Image Process. 2007; 6(1):29-52.

2. Bardaro C, Mantellini I. On convergence properties for a class of Kantorovich discrete operators. Numer Funct Anal Optim. 2012; 33(4):374-96.

3. Bardaro C, Musielak J, Vinti G. Nonlinear integral operators and applications. Gruyter Series in Nonlinear Analysis and Applications 9. New York - Berlin; 2003.

4. Costarelli D, Vinti G. Approximation by multivariate generalized sampling kantorovich operators in the setting of orlicz spaces, Bollettino U.M.I. (9) IV. 2011; 445-468.

5. Costarelli D, Vinti G. Approximation by nonlinear multivariate sampling-Kantorovich Type operators and applications to image processing. Numer Funct Anal Optim. 2013; 34(8):819-44.

6. Vinti G, Zampogni L. Approximation by means of nonlinear Kantorovich sampling type operators in Orlicz spaces. J. Approx. Theory. 2009; 161:511-28.

7. Vinti G, Zampogni L. A unifying approach to convergence of linear sampling type operators in Orlicz spaces. Adv Differential Equations. 2011; 16(5-6):573-600.

8. Cicelia JE. Mathematicals Method, Thermal Engineering. S\&S Publication; 2013. 